\title{
An Ab initio Study on the Convergence of Electronic Properties of SiC Nanotubes
}

\author{
Kapil Adhikari* and Asok K. Ray \\ Department of Physics, University of Texas at Arlington, Arlington, TX 76019 \\ Correspondence: kapila@uta.edu
}

\begin{abstract}
Ab initio calculations of the electronic structures of silicon carbide ( $\mathrm{SiC}$ ) nanotubes represented by clusters are presented. The nanotube clusters of chiralities $(3,3)$ and $(5,5)$ are studied using the hybrid density functional B3LYP (Becke's 3-parameter and the Lee-Yang-Parr exchange-correlation) and LANL2DZ (Los Alamos National Laboratory double $\zeta$ ) and 3-21G* basis sets. Evolution of electronic properties of silicon carbide $(\mathrm{SiC})$ nanotubes $(3,3)$ and $(5,5)$ with their length is discussed. The results suggest that the electronic properties of nanotubes change for short tubes of unit cells with lengths varying from 1 to 5. However, the properties do not seem to change significantly after this. Therefore, an infinite silicon carbide (SiC) nanotube can be approximated by a nanotube cluster of 5 unit cells.
\end{abstract}

Keywords: silicon carbide, nanotube clusters, hybrid density, unit cells.

\section{Introduction}

Discovery of multi-walled carbon nanotubes (CNT) in 1991[1] has led to an explosive growth of research interest in these kinds of quasi-one-dimensional structures. Carbon nanotubes have very interesting physical and electronic properties which make them very useful in electronics industry [2-8]. The properties of carbon nanotubes are strongly dependent on the diameter and chirality. Depending on their chirality, carbon nanotubes are either metallic or semiconductor which makes them useful for a wide range of applications. The strong dependence of electrical properties on chirality limits the application of carbon nanotubes in many nano-electronics applications because the semiconducting and metallic nanotubes are mixed in the sample of nanotubes grown in the lab. This involves an extra work for manufacturing electronic devices of carbon nanotubes because to obtain carbon nanotubes with similar electronic properties one has to do post synthesis separation of metallic and semiconducting nanotubes or control the synthesis of the nanotubes very precisely $[9,10]$. The strong need of materials which can be used even in harsh conditions has prompted a search for "better" alternatives to CNT. In search for "better" alternatives to CNT, a significant number of experimental and theoretical research works on nanostructures of other elements have been performed. A typical example is a nanotube formed by group-III nitrides. Group-III nitride nanotubes, such as $\mathrm{BN}, \mathrm{AlN}$, and $\mathrm{GaN}$, have been synthesized through different techniques [11-14]. Synthesis of several other nanotubes has been reported, for example, $\mathrm{NiCl}_{2}, \mathrm{H}_{2} \mathrm{Ti}_{3} \mathrm{O}_{3}, \mathrm{TiO}_{2}$, and $\mathrm{Si}$ [15-18]. Dai et al. [19] reported the synthesis of nanostructures by converting carbon nanotubes to carbide rods from reaction with volatile oxide and/or halide species. One of the nanorods produced was $\mathrm{SiC}$, among others such as $\mathrm{TiC}, \mathrm{NbC}, \mathrm{Fe}_{3} \mathrm{C}$, and $\mathrm{BC}_{\mathrm{x}}$. An interesting candidate here is silicon carbide ( $\mathrm{SiC}$ ) which, in bulk form, is one of the hardest materials and is very suitable for electronic devices designed for operations in extreme environments. Bulk silicon carbide has a wide band gap from $2.2 \mathrm{eV}$ for $3 \mathrm{C}-\mathrm{SiC}$ to $3.0 \mathrm{eV}$ for $6 \mathrm{H}-\mathrm{SiC}$, and to $3.3 \mathrm{eV}$ for $4 \mathrm{H}-\mathrm{SiC}[20]$. It has high thermal conductivity up to about $500 \mathrm{~W} /(\mathrm{mK})$ at room temperature [21] and thermal expansion coefficient smaller than $6 \mu / \mathrm{K}$ [22]. It has very high decomposition temperature of about $2545^{\circ} \mathrm{C}$ [23]. $3 \mathrm{C}-\mathrm{SiC}$ has very high Young's modulus 360-600 GPa depending upon its orientations [24].

In addition to the properties due to quantum size effects, silicon carbide nanostructures have some of these unique properties of bulk silicon carbide. Studies, in 
fact, have shown that silicon carbide nanostructures have additional unique properties compared to the bulk. $[25,26]$. Due to its partially ionic nature, SiCNTs have higher reactivity of the exterior surface than that of carbon nanotubes. This might be helpful to facilitate sidewall decoration.

Motivated by these extraordinary characteristics of bulk silicon carbide as well as silicon carbide nanowires, there has been numerous attempts, both theoretical and experimental, to study the possible graphitic phases of silicon carbide and possible applications in various fields. Most of the experimental attempts of fabricating SiCNTs showed the formation of $\beta$-SiC nanorods. Comparing the total energy of graphitic form with that of $\beta$-SiC according to calculation based on density functional theory, Miyamoto and $\mathrm{Yu}$ [27] have noted that $\mathrm{SiC}$ has a large difference between $\mathrm{sp}^{2}$ and $\mathrm{sp}^{3}$ bond structures with a value of $1.25 \mathrm{eV}$ per $\mathrm{Si}-\mathrm{C}$ pair. This energy difference makes the realization of graphitic phase of SiC very difficult. However, in the same study, Miyamoto and Yu have demonstrated the possibility of forming $\mathrm{SiC}$ nanotubes. They have reported that the strain energies of SiCNTs are lower than those of carbon nanotubes. They proposed the synthesis pathways by using an electronic technique of an extreme hole injection which would cause the graphitic sheet of $\mathrm{SiC}$ exfoliate from $\mathrm{SiC}$ (111) surface. Different groups have synthesized the SiCNTs using different techniques [28-36]. Sun et al. [38] have reported the synthesis of multi-walled $\mathrm{SiC}$ nanotubes through a substitutional reaction with $\mathrm{Si}$ atoms replacing half of the $\mathrm{C}$ atoms from a multiwalled carbon nanotube. The observed $\mathrm{SiC}$ nanotubes were multi-walled but with higher inter-planar spacing than those of multi-walled carbon nanotubes. Also the interlayer separation between the layers of nanotubes was found to be greater than $2.5 \AA$, which is typical for $\beta$-SiC. This indicated that the wall morphology of the observed nanotubes might correspond to graphitic phase rather than the cubic phase of the silicon carbide. Electron-energy-loss-spectroscopy (EELS) of the multi-walled SiCNTs has indicated possible $\pi$ bonding between $\mathrm{Si}$ and $\mathrm{C}$ atom. The interlayer separations of the multi-walled nanotubes were observed to be 3.5$4.5 \AA$. This indicated weak coupling between inner and outer tubes and the possibility of separating them with ease.

Most of the theoretical studies on SiCNTs are based on the graphene-like structure of silicon carbide. Although $\mathrm{SiC}$ graphitic monolayers have not yet been observed experimentally, some theoretical studies have predicted their possibilities. Also, the experimental observation by Sun et al. [36] has provided some evidences of graphitic phase of silicon carbide. The major driving force behind all the studies on SiCNTs is mainly their potential applications. Some ab initio methods [44] have shown that the most stable $\mathrm{SiC}$ nanotube has the ratio of $\mathrm{Si}$ to $\mathrm{C}$ of 1 to 1 . These studies claim that the nanotubes with other ratios will eventually collapse the tube into nanowires or clusters with solid interiors. Some other theoretical studies [38-41] have suggested that the stable silicon carbide nanotubes are of three types- types 1, 2 and 3, depending upon the atomic arrangement in the nanotube. Type 1 consists of alternating $\mathrm{Si}$ and $\mathrm{C}$ atoms with each $\mathrm{Si}$ atoms having three $\mathrm{C}$ neighbors and vice versa. In type 2 configuration, each $\mathrm{Si}$ atom has two $\mathrm{C}$ neighbors and one $\mathrm{Si}$ neighbor and vice versa. In type 3 , each $\mathrm{Si}$ has two $\mathrm{C}$ and one Si neighbors but $\mathrm{Si}$ and $\mathrm{C}$ atoms are arranged alternatively in each layer. In this work, we have considered the armchair $\mathrm{SiC}$ nanotubes in type 1 configuration. The evolution of the electronic properties of the $\mathrm{SiC}$ nanotubes with increase in length is presented.

\section{Construction of nanotube}

Similar to carbon nanotube, a SiCNT can be considered as a roll of silicon carbide graphene-like sheet. This rolling up can be described in terms of the chiral vector $\vec{C}_{h}$, which connects two sites of the two-dimensional graphene-like sheet that are crystallographically equivalent. This chiral vector maps an atom from the left hand border onto an atom on the right border line and is an integer multiple of the two basis vectors $\vec{a}_{1}$ and $\vec{a}_{2}$, i.e., $\vec{C}_{h}=n \vec{a}_{1}+m \vec{a}_{2}$. So the geometry of any nanotube can be described by the integer pair ( , $m$ ) which determines the chiral vector. An armchair nanotube corresponds to the case of $n=m$, and a zigzag nanotube corresponds to the case of $m=0$. All other $(n, m)$ chiral vectors correspond to chiral nanotubes. 
A nanotube is constructed by rolling a graphene sheet bounded by chiral vector $\vec{C}_{h}$ and translation vector $\vec{T}$ as shown in Figure 1. As an example, the figure has a shaded region bounded by chiral vector $\vec{C}_{h}=4 \vec{a}_{1}+2 \vec{a}_{2}$ and translation vector $\vec{T}$. When we roll the shaded region we get a nanotube with tube circumference $\left|\vec{C}_{h}\right|$ and tube length $|\vec{T}|$. The chiral indices of the nanotube in this example are therefore (4, 2 ). If we roll a nanotube along the dotted lines shown in figure, we get the zigzag or armchair nanotubes as indicated in the figure. In this letter we present the study of $\mathrm{SiC}$ nanotubes $(3,3)$ and $(5,5)$.

\section{Computational method and discussions of results}

In this work, hybrid density functional theory incorporating Hartree-Fock (HF) exchange [42, 43, 44] with density functional theory (DFT) exchangecorrelation [45] has been used to study the electronic and geometric structure properties of the SiC SWNTs. In particular, we have used the B3LYP hybrid functional [46-49] and two basis sets namely - the all electron 3-21G* basis set [44], and the Los Alamos National Laboratory double $\zeta$ (LANL2DZ) basis set [50] as implemented in the GAUSSIAN 09 suite of programs [51] for full geometry optimizations without any symmetry constraints of the nanotube structures. Hybrid functionals are in general found to be efficient in reproducing the band gaps of semiconductors and insulators $[52,53]$ by treating the exchange part of the interactions better. We note here that, though different DFT functionals may produce slightly different quantitatively but not qualitatively different results, studies on semi-conducting materials have shown that, hybrid functionals, in particular B3LYP, is one of the most efficient and computationally inexpensive among all the DFT functionals available for calculation of electronic and structural properties of the semiconducting materials. All computations reported here have been performed at the supercomputing facilities of the University of Texas at Arlington.

Nanotubes are constructed as described in the previous section. The dangling bonds at the end of nanotubes are saturated with hydrogen atom. Length of nanotubes $(3,3)$ and $(5,5)$ is gradually increased by increasing the number of unit cells of the nanotubes. The purpose of this work is to study the convergence of electronic properties of $\mathrm{SiC}$ nanotubes with length. Figure 2 shows side and top views of the $\mathrm{SiC}$ nanotubes $(3,3)$ and $(5,5)$ with length equal to five unit cells.

To check the stability of the nanotubes, the cohesive energy or the binding energy per atom of each nanotube is calculated from:

$\mathrm{E}_{\mathrm{b}}=\left[\mathrm{aE}(\mathrm{Si})+\mathrm{bE}(\mathrm{C})+\mathrm{cE}(\mathrm{H})-\mathrm{E}\left(\mathrm{Si}_{\mathrm{a}} \mathrm{C}_{\mathrm{b}} \mathrm{H}_{\mathrm{c}}\right)\right] /(\mathrm{a}+\mathrm{b}+\mathrm{c})$ (1)

where $\mathrm{a}, \mathrm{b}$ and $\mathrm{c}$ are the numbers of $\mathrm{Si}, \mathrm{C}$, and $\mathrm{H}$ atoms respectively and $\mathrm{E}\left(\mathrm{Si}_{\mathrm{a}} \mathrm{C}_{\mathrm{b}} \mathrm{H}_{\mathrm{c}}\right)$ is the total energy of the clusters representing the nanotubes.

Tables 1 and 2 and figure 3 show the variation binding energy per atom of nanotubes $(3,3)$ and $(5,5)$ with the number of unit cells. It is evident that, as we increase the length of nanotubes binding energy increases. However, the rate of increment in binding energy with length is not very significant after a few number of unit cells. For example, the binding energy of nanotube $(3,3)$ increases by more than $10 \%$ from unit cell 1 to unit cell 2 whereas the increment is merely $1 \%$ from unit cell 5 to unit cell 6 . This indicates that the stability of a silicon carbide nanotube reaches saturation at about five unit cells. Given the same number of unit cells, the binding energy of nanotube $(3,3)$ is consistently smaller than that of $(5,5)$. Diameter of nanotube $(3,3)$ is $5.20 \AA$, and that of $(5,5)$ is $8.63 \AA$. The result therefore suggests that the nanotubes with greater diameter are more stable than those with the smaller diameter, given the same tube length. This has been confirmed by all the ab initio studies of such binary nanotubes [54].

The energy differences between the highest occupied molecular orbital (HOMO) and the lowest unoccupied molecular orbital (LUMO) give a measure of the "band gap" for the infinite nanotubes. This is approximate because the tubes studied here are "finite" in length, apart from the fact that hybrid density functional B3LYP tends to slightly over estimate energy gap [38]. Also, the purpose of this study is to determine the optimum length of a silicon carbide nanotube which can reasonably represent infinite nanotube. This 
measure is at least qualitatively correct to compare within a given type of nanotubes with different diameters, and this is sufficient for our present discussions. Tables 1, 2 and figure 4 show the variation of HOMO-LUMO gap with length of the nanotubes. It is clear from the figure that the gap changes with length of the nanotube and stabilizes after about 5 . The gap does not change significantly after 5 unit cells. The density of state (DOS) plot of $\mathrm{SiC}$ nanotubes $(3,3)$ and $(5,5)$ with length equal to 5 and 10 unit cells is shown in figure 5. Figure 6 shows the HOMOLUMO plot of the nanotubes $(3,3)$ and $(5,5)$ with 5 and 10 unit cells. Both these figures suggest that there is no significant difference in electronic characteristics of the nanotubes along the direction of tube axis (increasing number of unit cells). However, along the circumferential direction (changing the chirality) the electronic properties change. Mulliken population analysis of the nanotubes shows that the silicon atoms lose charge and carbon atoms gain charge. The charge transfer between the atoms is $0.90 e$ in $\mathrm{SiC}$ nanotube $(3,3)$ irrespective of its length. The charge transfer is 0.96 e in $\mathrm{SiC}$ nanotube $(5,5)$, irrespective of its length. The increased ionicity of the bond in $(5,5)$ is the reason why it has more HOMO-LUMO gap than $(3,3)$. The curvature of the nanotube suppresses the ionicity of the bond in $(3,3)$. A detailed analysis of the effect of curvature on the ionicity and hence the band gap of the nanotube is presented in our previous work [54].

\section{Conclusion}

We have presented a study on evolution of electronic properties and stability of silicon carbide nanotubes along the nanotube axis. The results suggest that although the properties of a nanotube changes with its length for very short nanotubes of 1-5 unit cells, they do not change significantly after 5 unit cells. Hence an infinite $\mathrm{SiC}$ nanotube can be approximated by a nanotube of length 5 unit cells. Also, this result confirms that the properties of a nanotube are continuous along the axial direction while they are quantized along the circumferential direction.

\section{References}

[1] S. Iijima, Nature 354 (1991) 56-58. doi: 10.1038/354056a0.

[2] S. Iijima and T. Ichihashi, Nature 363 (1993) 603605. doi: $10.1038 / 363603 \mathrm{a} 0$.

[3] M. S. Dresselhaus, G. Dresselhaus, and Ph. Avouris, Topics in Applied Physics, Vol. 80, Springer, Berlin, (2001).

[4] N. Hamada, S. I. Sawada, and A. Oshiyama, Phys. Rev. Lett. 68 (1992) 1579-1581. doi:10.1103/ PhysRevLett.68.1579.

[5] R. Saito, et al., Appl. Phys. Lett. 60(1992) 22042206. doi: $10.1063 / 1.107080$.

[6] T. W. Odom, et al., Nature 391 (1998) 62-64. doi:10.1038/34145.

[7] R. A. Jishi, J. Bragin, and L. Lou, Phys. Rev. B 59 (1999) 9862-9865. doi:10.1103/PhysRevB.59.9862.

[8] O. Gulseren, T. Yildirim, and S. Ciraci, Phys. Rev. B, 65 (2002), 153405-1-4. doi:10.1103/ PhysRevB.65.153405.

[9] R. Krupke, et al., Science 301, 344-347 (2003). doi: 10.1126/science.1086534.

[11] Y. Maeda, et al., J. Am. Chem. Soc. 127 10287-10290 (2005). doi: 10.1021/ja051774o.

[12] J. Cumings, and A. Zettl, Chem. Phys. Lett. 316(2000) 211-216. doi:10.1016/S0009-2614(99)01277-4.

[13] Q. Wu, et al., J. Am. Chem. Soc. 125 (2003) 10176-7. doi: $10.1021 / \mathrm{ja} 0359963$.

[14] J. Goldberger, et al., Nature 422, 599-602 (2003). doi: 10.1038/nature01551.

[15] Y. R. Hacohen, et al., Nature 395, 336-337 (1998). doi: $10.1038 / 26380$.

[16] Q. Chen, et al., Adv. Mater. 14, 1208$1211 \quad$ (2002). doi:10.1002/1521$4095(20020903) 14: 17<1208:$ : A ID ADMA1208>3.0.CO;2-0.

[17] G. R. Patzke, F. Krumeich, and R. Nesper, Ang. Chem. (Internationaled.inEnglish)41,2446-2461(2002). doi: 10.1002/1521-3773(20020715)41:14<2446::AIDANIE2446 $>3.0 . \mathrm{CO} ; 2-\mathrm{K}$.

[18] J. Sha, et al., Adv. Mater. 14, 1219-1221 (2002). doi: 10.1002/1521-4095(20020903)14:17<1219::AIDADMA1219>3.0.CO;2-T.

[19] H. Dai, et al., Nature 375, 769-772 (1995). doi: $10.1038 / 375769 \mathrm{a} 0$.

[20] J. Luning, et al., Phys. Rev. B 59, 10573-10582 (1999). doi:10.1103/PhysRevB.59.10573.

[21] J. Li, L. Porter, and S. Yip, J. Nucl. Mater. 255, 139152 (1998). doi: 10.1016/S0022-3115(98)00034-8.

[22] L. Porter, J. Li, and S. Yip, J. Nucl. Mater. 246, 5359. doi: 10.1016/S0022-3115(97)00035-4.

[23] R.W. Olesinski and G.J. Abbaschian, Bull. Alloy Phase Diag. 5, 486-489 (1984). doi:10.1007/ 
BF02872902.

[24] W.R.L. Lambrecht, et al., Phys. Rev. B 44, 3685 3694 (1991). doi:10.1103/PhysRevB.44.3685.

[25] E. W. Wong, P. E. Sheehan, and C. M. Lieber, Science 277, 1971-1975 (1997). doi:10.1126/ science.277.5334.1971.

[26] W. Han, et al., Chem. Phys. Lett. 265, 374-378 (1996). doi:10.1016/S0009-2614(96)01441-8.

[27] Y. Miyamoto and B. D. Yu, Appl. Phys. Lett. 80, 586588 (2002). doi:10.1063/1.1445474.

[28] C. Pham-Huu, N. Keller, and G. Ehet, J. Catal. 200, 400-410 (2001). doi:10.1006/jcat.2001.3216.

[29] E. Borowiak-Palen, et al., J. Appl. Phys. 97, $056102-$ 1-3 (2005). doi:10.1063/1.1853493.

[30] J. Q. Hu, et al., Appl. Phys. Lett. 85, 2932-2934 (2004). doi:10.1063/1.1801168.

[31] N. Keller, et al., Carbon 41, 2131-2139 (2003). doi:10.1016/S0008-6223(03)00239-2.

[32] T. Taguchi, et al., Physica E 28, 431-438 (2005). doi:10.1016/j.physe.2005.05.048.

[33] J. M. Nhut, et al., Catal. Today 76, 11-32 (2002). doi:10.1016/S0920-5861(02)00206-7.

[34] T. Taguchi, et al., J. Am. Ceram. Soc. 88, 459-461 (2005). doi:10.1111/j.1551-2916.2005.00066.x.

[35] A. Huczko, et al., J. Phy. Chem. B, 109, 16244-16251 (2005). doi:10.1021/jp050837m.

[36] X. H. Sun, et al., J. Am. Chem. Soc. 124, $14464-$ 14471 (2002). doi:10.1021/ja0273997.

[37] A. Mavrandonakis, et al., Nano Lett. 3, 1481-1484 (2003). doi:10.1021/n10343250.

[38] M. Menon, et al., Phys. Rev. B 69, 45-48 (2004). doi:10.1103/PhysRevB.69.115322.

[39] K. Alam and A. K. Ray, Phys. Rev. B 77, 035436-110 (2008). doi:10.1103/PhysRevB.77.035436.

[40] K. Adhikari and A. K. Ray, Sol. St. Comm. 151, 430435 (2011). doi: 10.1016/j.ssc.2011.01.004.

[41] K. Adhikari and A. K. Ray, E. Phys. J. D 64, 353-363 (2011). doi: 10.1140/epjd/e2011-20280-3.

[42] D. R. Hartree, Proc. Cam. Phil. Soc. 24, 89-110 (1928). doi:10.1017/S0305004100011919.

[43] V. Fock, Z. Phys. 61, 126-148 (1930). doi:10.1007/ BF01340294.

[44] W.J. Hehre, et al., Ab initio Molecular Orbital Theory, first ed., Wiley, New York, 1986.

[45] J.P. Perdew, et al. Phys. Rev. Lett. 49, 1691-1694 (1982). doi:10.1103/PhysRevLett.49.1691.

[46] S. H. Vosko, L. Wilk and M. Nusair, Can. J. Phys. 58, 1200-1211 (1980). doi: 10.1139/p80-159.

[47] A.D. Becke, J. Chem. Phys. 98, 5648-5652 (1993). doi:10.1063/1.464913.

[48] C. Lee, W. Yang, R.G. Parr, Phys. Rev. B 37, 785-789 (1988). doi:10.1103/PhysRevB.37.785.

[49] P.J. Stephens, et al., J. Phys. Chem. 98, 11623-11627
(1994). doi: 10.1021/j100096a001.

[50] P.J. Hay and W.R. Wadt, J. Chem. Phys. 82, 270-283 (1985). doi:10.1063/1.448799.

[51] M. J. Frisch, et al., Gaussian 09, Gaussian, Inc., Wallingford CT, 2009.

[52] J. Muscat, A. Wander, N. M. Harrison, Chem. Phys. Lett. 342, 397-401 (2001). doi:10.1016/S00092614(01)00616-9.

[53] J. Heyd, and G. Scuseria, J. Chem. Phys. 121, $1187-$ 1192 (2004). doi:10.1063/1.1760074.

[54] K. Adhikari, M. N. Huda and A. K. Ray, J. Comp. Theo. Nano. 8, 1502-1508 (2011). doi:10.1166/ jetn.2011.1842.

Table 1: Binding energy per atom $(\mathrm{eV})$ and HOMO-LUMO gap $(\mathrm{eV})$ of $\mathrm{SiC}$ nanotube clusters $(3,3)$ calculated using basis sets LANL2DZ and 3-21G*.

\begin{tabular}{|c|l|l|l|l|l|}
\hline \multirow{2}{*}{$\begin{array}{c}\text { No. of unit } \\
\text { cells }\end{array}$} & \multirow{2}{*}{$\begin{array}{c}\text { Stoichiom- } \\
\text { etry }\end{array}$} & \multicolumn{2}{|c|}{ B.E./atom(eV) } & \multicolumn{2}{c|}{$\begin{array}{c}\text { HOMO-LUMO } \\
\text { gap(eV) }\end{array}$} \\
\cline { 3 - 6 } & & $3-21 \mathrm{G}^{*}$ & $\begin{array}{c}\text { LANL } \\
2 \mathrm{DZ}\end{array}$ & $3-21 \mathrm{G}^{*}$ & $\begin{array}{c}\text { LAN- } \\
\text { L2DZ }\end{array}$ \\
\hline 1 & $\mathrm{Si}_{6} \mathrm{C}_{6} \mathrm{H}_{12}$ & 3.822 & 3.621 & 4.346 & 4.244 \\
\hline 2 & $\mathrm{Si}_{12} \mathrm{C}_{12} \mathrm{H}_{12}$ & 4.342 & 4.003 & 3.681 & 2.977 \\
\hline 3 & $\mathrm{Si}_{18} \mathrm{C}_{18} \mathrm{H}_{12}$ & 4.606 & 4.202 & 3.435 & 2.872 \\
\hline 4 & $\mathrm{Si}_{24} \mathrm{C}_{24} \mathrm{H}_{12}$ & 4.764 & 4.320 & 3.408 & 2.868 \\
\hline 5 & $\mathrm{Si}_{30} \mathrm{C}_{30} \mathrm{H}_{12}$ & 4.869 & 4.399 & 3.335 & 2.769 \\
\hline 6 & $\mathrm{Si}_{36} \mathrm{C}_{36} \mathrm{H}_{12}$ & 4.945 & 4.455 & 3.352 & 2.750 \\
\hline 7 & $\mathrm{Si}_{42} \mathrm{C}_{42} \mathrm{H}_{12}$ & 5.001 & 4.497 & 3.289 & 2.677 \\
\hline 8 & $\mathrm{Si}_{48} \mathrm{C}_{48} \mathrm{H}_{12}$ & 5.045 & 4.530 & 3.293 & 2.678 \\
\hline 9 & $\mathrm{Si}_{54} \mathrm{C}_{54} \mathrm{H}_{12}$ & 5.080 & 4.557 & 3.262 & 2.621 \\
\hline 10 & $\mathrm{Si}_{60} \mathrm{C}_{60} \mathrm{H}_{12}$ & 5.109 & 4.578 & 3.254 & 2.614 \\
\hline
\end{tabular}

Table 2: Binding energy per atom (eV) and HOMO-LUMO gap $(\mathrm{eV})$ of $\mathrm{SiC}$ nanotube clusters $(5,5)$ calculated using basis sets LANL2DZ and $3-21 \mathrm{G}^{*}$.

\begin{tabular}{|c|c|c|c|c|c|}
\hline \multirow{2}{*}{$\begin{array}{c}\text { No. of unit } \\
\text { cells }\end{array}$} & \multirow{2}{*}{ Stoichiometry } & \multicolumn{2}{|c|}{ B.E./atom(eV) } & \multicolumn{2}{|c|}{$\begin{array}{c}\text { HOMO-LUMO } \\
\text { gap(eV) }\end{array}$} \\
\cline { 3 - 6 } & $3-21 \mathrm{G}^{*}$ & $\begin{array}{c}\text { LAN- } \\
\text { L2DZ }\end{array}$ & $3-21 \mathrm{G}^{*}$ & $\begin{array}{c}\text { LAN- } \\
\text { L2DZ }\end{array}$ \\
\hline 1 & $\mathrm{Si}_{10} \mathrm{C}_{10} \mathrm{H}_{20}$ & 3.859 & 3.656 & 3.138 & 2.620 \\
\hline 2 & $\mathrm{Si}_{20} \mathrm{C}_{20} \mathrm{H}_{20}$ & 4.424 & 4.102 & 3.805 & 3.229 \\
\hline 3 & $\mathrm{Si}_{30} \mathrm{C}_{30} \mathrm{H}_{20}$ & 4.710 & 4.327 & 3.437 & 2.965 \\
\hline 4 & $\mathrm{Si}_{40} \mathrm{C}_{40} \mathrm{H}_{20}$ & 4.880 & 4.462 & 3.434 & 2.978 \\
\hline 5 & $\mathrm{Si}_{50} \mathrm{C}_{50} \mathrm{H}_{20}$ & 4.994 & 4.551 & 3.541 & 3.094 \\
\hline 6 & $\mathrm{Si}_{60} \mathrm{C}_{60} \mathrm{H}_{20}$ & 5.075 & 4.615 & 3.469 & 2.998 \\
\hline 7 & $\mathrm{Si}_{70} \mathrm{C}_{70} \mathrm{H}_{20}$ & 5.135 & 4.663 & 3.486 & 3.031 \\
\hline 8 & $\mathrm{Si}_{80} \mathrm{C}_{80} \mathrm{H}_{20}$ & 5.183 & 4.701 & 3.476 & 3.055 \\
\hline 9 & $\mathrm{Si}_{90} \mathrm{C}_{90} \mathrm{H}_{20}$ & 5.220 & 4.730 & 3.470 & 3.027 \\
\hline 10 & $\mathrm{Si}_{100} \mathrm{C}_{100} \mathrm{H}_{20}$ & 5.252 & 4.755 & 3.472 & 3.055 \\
\hline
\end{tabular}

